\title{
On feedback stabilisation of switched discrete-time systems via Lie-algebraic techniques
}

\author{
Hernan Haimovich, Julio H. Braslavsky and Flavia Felicioni
}

\begin{abstract}
This paper studies the stabilisation of switched discrete-time linear control systems under arbitrary switching. A sufficient condition for the uniform global exponential stability (UGES) of such systems is the existence of a common quadratic Lyapunov function (CQLF) for the component subsystems. The existence of such CQLF can be ensured using Lie-algebraic techniques by the existence of a nonsingular similarity transformation that simultaneously triangularises the closed-loop evolution maps of the component subsystems. The present work formulates a Lie-algebraic feedback design problem in terms of invariant subspaces and proposes an iterative algorithm that seeks a set of feedback maps that guarantee the existence of a CQLF, and thus UGES of the switched feedback system. The main contribution of the paper is to show that this algorithm will find the required feedback maps if and only if the Lie-algebraic problem has a solution.

Index Terms-Switched systems, Lyapunov methods, closed loop systems, asymptotic stability, Lie algebras.
\end{abstract}

\section{INTRODUCTION}

A switched system is a hybrid system that can be mathematically described by a set of indexed differential or difference equations that are switched according to some logical rule or regime. Such systems arise in many diverse areas of application, such as the regulation of complex dynamical processes [18], and the control of data flow in large information networks [10].

In recent years, the study of stability of switched systems has attracted increasing research activity. A number of survey papers [14], [4], [20], [16], and books [19], [12] summarise many important advances in the area.

This paper considers the problem of asymptotic stabilisation of switched discrete-time control systems under arbitrary switching regimes. It is well-known that the UGES of a switched system under arbitrary switching is equivalent to the existence of a common Lyapunov function for the component subsystems [17]. Finding such a common Lyapunov function is not easy in general, and thus, much research activity focused on studying the existence of a CQLF [20, §4.2].

Quadratic Lyapunov functions are appealing because they can be constructed easily for linear systems, and they lend themselves to efficient numerical computation by solving linear matrix inequalities (LMIs) [15]. The application of LMIs to find or check for the existence of a CQLF, however,

H. Haimovich and F. Felicioni are with CONICET and Laboratorio de Sistemas Dinámicos y Procesamiento de Información, Departamento de Control, Facultad de Cs. Exactas, Ingeniería y Agrimensura, Universidad Nacional de Rosario, Riobamba 245bis, 2000 Rosario, Argentina. h.haimovich@gmail.com, flaviafefceia.unr.edu.ar

J. H. Braslavsky is with the ARC Centre of Excellence for Complex Dynamic Systems \& Control, The University of Newcastle, Australia. julio.braslavsky@newcastle.edu.au has two main drawbacks: (1) numerical complexity increases rapidly as dimensions grow, and (2), little theoretical insight is gained into the structural obstructions to the existence of a CQLF for a given system [20, §4.3], [15], [16].

An elegant conceptual framework to analyse the stability of switched systems is based on Lie-algebraic conditions [13], [1], [9], [22]. The Lie-algebraic approach offers valuable structural insight into the stability problem as well as some computational advantages [13]. For switched discretetime systems, the existence of a CQLF is guaranteed if, in addition to the stability of each of the component subsystems, the Lie algebra generated by their evolution operators is solvable. Such Lie-algebraic condition is more restrictive than the existence of a CQLF. A more general condition is available for switched continuous-time systems [1].

The above Lie-algebraic conditions apply to autonomous systems under arbitrary switching. The present paper applies similar Lie-algebraic techniques to analyse the stabilisability of switched discrete-time linear systems with control inputs. We study the design of a set of stabilising feedback control laws that also satisfy the Lie-algebraic conditions that guarantee the existence of a CQLF. This feedback stabilisation problem is formulated in the geometric approach of Wonham [23] and Basile and Marro [3]. In this way, solving the problem is equivalent to finding a set of state feedback maps that, in addition to ensuring the stability of the component subsystems, render invariant a flag of subspaces (to be defined in Section II) simultaneously under each of the closed-loop maps of the component subsystems.

The feedback stabilisation of discrete-time switched linear systems using Lie algebraic techniques has been addressed in [7], [6], [8]. The latter works consider the specific case of switched discrete-time systems arising from continuoustime systems sampled at a varying rate, but where at each sampling instant the subsequent sampling period can only be selected from a finite set of known values. In [7], control design for this type of system is pursued and achieved for some special cases: (i) pairwise commuting closed-loop system matrices, (ii) simultaneous triangularisation, where the triangularising transformation can be directly obtained from the open-loop continuous-time system matrix, and (iii) second order systems. In [6], the results of [7] are enhanced by analysing robustness and performance taking consideration of the fact that a diagonal CQLF always exists for stable subsystems in triangular form. In [8], control design through iterative common eigenvector assignment with stability is suggested as a possible control and observer design methodology, although conditions to show that the 
methodology itself is not restrictive are not given, nor a procedure for its numerical implementation.

The present paper extends the ideas in [7], [6], [8] in two main directions. First, it deals with general switched discrete-time linear systems and not just those arising from sampling a single continuous-time system. Second, and most importantly, necessary and sufficient conditions indicating the success or otherwise of the methodology are derived. We propose an algorithm that seeks a solution iteratively, by simultaneous assignment of a common eigenvector with corresponding stable eigenvalues. Our main result establishes that this iterative algorithm will find the required feedback maps in a finite number of steps if and only if the problem has a solution. The main significance of our result is its necessity part. Sufficiency follows straightforwardly from typical Lie-algebraic considerations used in autonomous systems. Necessity, however involves explicit consideration of the fact that control inputs are present.

The rest of the paper is organised as follows. In Section II we more precisely define the control design problem considered, and introduce notation and some concepts required in Section III to describe the proposed iterative algorithm that solves this problem whenever a solution exists. Our main result is stated and discussed in Section III-A, and proved in Section III-B (with some auxiliary results proved in the Appendix). Section IV presents the paper conclusions.

\section{Notation}

The spectrum (set of eigenvalues) of a square matrix $A$ is denoted $\boldsymbol{\sigma}(A)$, and the spectral radius of $A$ is denoted $\boldsymbol{\rho}(A)$. Calligraphic upper case letters $(\mathcal{X}, \mathcal{V}, \mathcal{S})$ denote vector spaces, and $d(\mathcal{X})$ denotes the dimension of a vector space $\mathcal{X}$. If $\mathcal{X}$ is a vector space, then $\mathcal{S} \subset \mathcal{X}$ means that $\mathcal{S}$ is a subspace of $\mathcal{X}$ and $\mathcal{X} / \mathcal{S}$ denotes the quotient space. A linear map is denoted as $A: \mathcal{X} \rightarrow \mathcal{Y}$, and if $A: \mathcal{X} \rightarrow \mathcal{X}, \mathcal{S} \subset \mathcal{X}$, and $A \mathcal{S} \subset \mathcal{S}$, then $A \mid \mathcal{S}$ denotes the restriction of $A$ to $\mathcal{S}$ with codomain $\mathcal{S}$. The kernel (null space) of a linear map $A$ is denoted by ker $A$. Depending on the context, 0 denotes $0 \in \mathbb{R}, 0 \in \mathbb{C}$, the zero vector, zero map, or zero-dimensional vector space. If $L$ and $M$ are two sets, then $L \backslash M$ denotes the set $\{x \in L: x \notin M\}$. We refer to [23, $\S 0]$ for other standard linear algebra concepts and notation used.

\section{PROBLEM FORMULATION}

\section{A. Discrete-time switched control systems}

Consider the linear discrete-time switched system (DTSS)

$$
x_{k+1}=A_{i(k)} x_{k}+B_{i(k)} u_{k},
$$

where $x_{k} \in \mathbb{R}^{n}, u_{k} \in \mathbb{R}^{m}$,

$$
i(k) \in\{1,2, \ldots, N\}, \quad \text { for all } k,
$$

and the matrices $A_{1}, \ldots, A_{N} \in \mathbb{R}^{n \times n}$ and $B_{1}, \ldots, B_{N} \in$ $\mathbb{R}^{n \times m}$ are known. We are interested in state-feedback control design of the form

$$
u_{k}=K_{i(k)} x_{k}
$$

so that the resulting closed-loop system

$$
\begin{aligned}
x_{k+1} & =A_{i(k)}^{\mathrm{CL}} x_{k}, \quad \text { where } \\
A_{i}^{\mathrm{CL}} & =A_{i}+B_{i} K_{i}, \quad \text { for } i=1, \ldots, N,
\end{aligned}
$$

be exponentially stable for arbitrary switching. Note that at every time instant $k$, the control law (3) requires knowledge of the "active" subsystem given by $i(k)$.

As is well-known, ensuring that $\rho\left(A_{i}^{\mathrm{CL}}\right)<1$ for $i=$ $1, \ldots, N$ is necessary but not sufficient to ensure the stability of the DTSS (1) for arbitrary switching. A sufficient condition is given by the following result, which is a minor modification of [22, Theorem 6.18].

Lemma 1 If $\boldsymbol{\rho}\left(A_{i}^{\mathrm{CL}}\right)<1$ for $i=1, \ldots, N$, and the Lie algebra generated by $\left\{A_{i}^{\mathrm{CL}}: i=1, \ldots, N\right\}$ is solvable, then (4) admits a common quadratic Lyapunov function and hence is exponentially stable.

In this paper, we specifically consider stabilising state feedback design based on the Lie-algebraic condition of Lemma 1. In matrix terms, the fact that the Lie algebra generated by the matrices $A_{i}^{\mathrm{CL}}$ is solvable is equivalent to the existence of an invertible matrix $T \in \mathbb{C}^{n \times n}$ such that $T^{-1} A_{i}^{\mathrm{CL}} T$ is upper triangular for $i=1, \ldots, N$. That is, each matrix $A_{i}^{\mathrm{CL}}$ for $i=1, \ldots, N$ is similar to an upper triangular matrix under a common similarity transformation $T$. Note that even if the matrices $A_{i}^{\mathrm{CL}}$ have real entries, those of the transformation $T$ may be complex [5].

\section{B. Invariant subspaces}

The requirement that a matrix $A_{i}^{\mathrm{CL}} \in \mathbb{R}^{n \times n}$ be similar to an upper triangular matrix can be posed in geometric terms as follows. Let $\mathcal{X}$ denote the state space, which, even if $A_{i}^{\mathrm{CL}}: \mathbb{R}^{n} \rightarrow \mathbb{R}^{n}$ necessarily has to be taken as $\mathbb{C}^{n}$ (the complexification of $\mathbb{R}^{n}$, see [23]), and consider the corresponding map $A_{i}^{\mathrm{CL}}: \mathcal{X} \rightarrow \mathcal{X}$. The following definitions will be used in the sequel.

Definition 1 (Flag, invariant flag) A chain of subspaces of $\mathcal{X}$ satisfying

$$
\begin{aligned}
\mathcal{S}_{1} \subset \mathcal{S}_{2} \subset \cdots \subset & \mathcal{S}_{n}=\mathcal{X}, \\
& \text { where } d\left(\mathcal{S}_{j}\right)=j, \text { for } j=1,2, \ldots, n,
\end{aligned}
$$

is called a flag of $\mathcal{X}$. If each subspace $\mathcal{S}_{j}$ in the flag is invariant with respect to some map $A: \mathcal{X} \rightarrow \mathcal{X}$, namely,

$$
A \mathcal{S}_{j} \subset \mathcal{S}_{j}, \quad \text { for } j=1,2, \ldots, n,
$$

then the flag is said to be invariant ${ }^{1}$ under the map $A$.

The motivation for these definitions is that if there exist a flag of subspaces $\left\{\mathcal{S}_{j}, j=1, \ldots, n\right\}$ that is simultaneously invariant under the closed-loop maps $A_{i}^{\mathrm{CL}}$ for $i=1, \ldots, N$, then in a basis $\left\{s_{1}, \ldots, s_{n}\right\}$ for $\mathcal{X}$, where $\left\{s_{1}, \ldots, s_{j}\right\}$ is

\footnotetext{
${ }^{1}$ In Algebra, such $A$ is sometimes said to "stabilise" the flag (see, for example, $[11, \S 3.3])$. However, we reserve the term stabilisation to refer to the concept typically used in Systems and Control.
} 
a basis for $\mathcal{S}_{j}$, the maps $A_{i}^{\mathrm{CL}}$ are all represented by upper triangular matrices. By Lie's Theorem [11, $\S 4.1$, Corollary $\mathrm{A}]$, the existence of a flag in $\mathcal{X}$ that is invariant under all matrices $A_{i}^{\mathrm{CL}}, i=1, \ldots, N$ is equivalent to the Lie-algebraic condition of Lemma 1 . We may thus formulate the problem to be addressed as follows.

Problem 1 Given the maps $A_{i}: \mathbb{R}^{n} \rightarrow \mathbb{R}^{n}$ and $B_{i}: \mathbb{R}^{m} \rightarrow$ $\mathbb{R}^{n}$ for $i=1,2, \ldots, N$, find maps $K_{i}: \mathbb{R}^{n} \rightarrow \mathbb{R}^{m}$ such that $\boldsymbol{\rho}\left(A_{i}^{\mathrm{CL}}\right)<1$ with $A_{i}^{\mathrm{CL}}$ as in (5), and such that there exists a flag of $\mathcal{X}=\mathbb{C}^{n}$,

$$
\begin{aligned}
\mathcal{S}_{1} \subset \mathcal{S}_{2} \subset \cdots & \subset \mathcal{S}_{n}=\mathcal{X}, \\
& \text { where } d\left(\mathcal{S}_{j}\right)=j, \text { for } j=1,2, \ldots, n,
\end{aligned}
$$

that is invariant under each $A_{i}^{\mathrm{CL}}$,

$$
A_{i}^{\mathrm{CL}} \mathcal{S}_{j} \subset \mathcal{S}_{j}, \quad \text { for } j=1,2, \ldots, n .
$$

If the feedback maps $K_{i}$ solving Problem 1 exist, they guarantee the existence of a CQLF for the closed-loop maps $A_{i}^{\text {CL }}$. Because these closed-loop maps will all be triangular in a basis $\left\{s_{1}, \ldots, s_{n}\right\}$ of $\mathcal{X}$, a (diagonal) CQLF can be computed [22]. Thus, a solution to Problem 1 provides a way to find a common quadratic control Lyapunov function (in the sense of [2], [21]) for the switched system.

\section{Stabilising Feedback Control Design}

\section{A. Control Design Algorithm}

This section presents the main result of the paper, Theorem 1, which shows that Problem 1 can be solved if and only if an iterative algorithm terminates successfully. At each iteration, this algorithm seeks feedback maps so that a set of closed-loop maps have a common eigenvector and, for each of these closed-loop maps, the eigenvalue corresponding to the common eigenvector is stable. The fact that Problem 1 is solved if the algorithm successfully terminates follows easily from standard Lie-algebraic considerations. The converse implication, however, is much more involved and constitutes the core contribution of the paper. The aforementioned algorithm is given in pseudocode as Algorithm 1.

The common eigenvector assignment procedure (Procedure 1) executed at each step of Algorithm 1 is the following.

Procedure 1 (Common eigenvector assignment with stability) Given the maps $A_{i}^{\ell}: \mathcal{X}_{\ell} \rightarrow \mathcal{X}_{\ell}$ and $B_{i}^{\ell}: \mathcal{U} \rightarrow \mathcal{X}_{\ell}$, for $i=1, \ldots, N$, compute, if possible, a set of feedback maps $F_{i}^{\ell}: \mathcal{X}_{\ell} \rightarrow \mathcal{U}$ and a one-dimensional subspace $\mathcal{V}_{\ell}$ such that for $i=1, \ldots, N$,

$$
\begin{gathered}
\left(A_{i}^{\ell}+B_{i}^{\ell} F_{i}^{\ell}\right) \mathcal{V}_{\ell} \subset \mathcal{V}_{\ell} \\
\boldsymbol{\rho}\left(A_{i}^{\ell}+B_{i}^{\ell} F_{i}^{\ell} \mid \mathcal{V}_{\ell}\right)<1
\end{gathered}
$$

Also, if $A_{i}^{\ell}$ and $B_{i}^{\ell}$ are real, then $F_{i}^{\ell}$ will be real. If, in addition, $\mathcal{V}_{\ell}$ is complex, then $F_{i}^{\ell+1}=0$.

The condition $F_{i}^{\ell+1}=0$ in the case $A_{i}^{\ell}$ and $B_{i}^{\ell}$ are real (and hence so are $F_{i}^{\ell}$ ) with $\mathcal{V}_{\ell}$ complex is only ensuring that if a complex eigenvector is assigned, its complex conjugate will be assigned as well, which will ultimately ensure that the feedback maps sought are real.

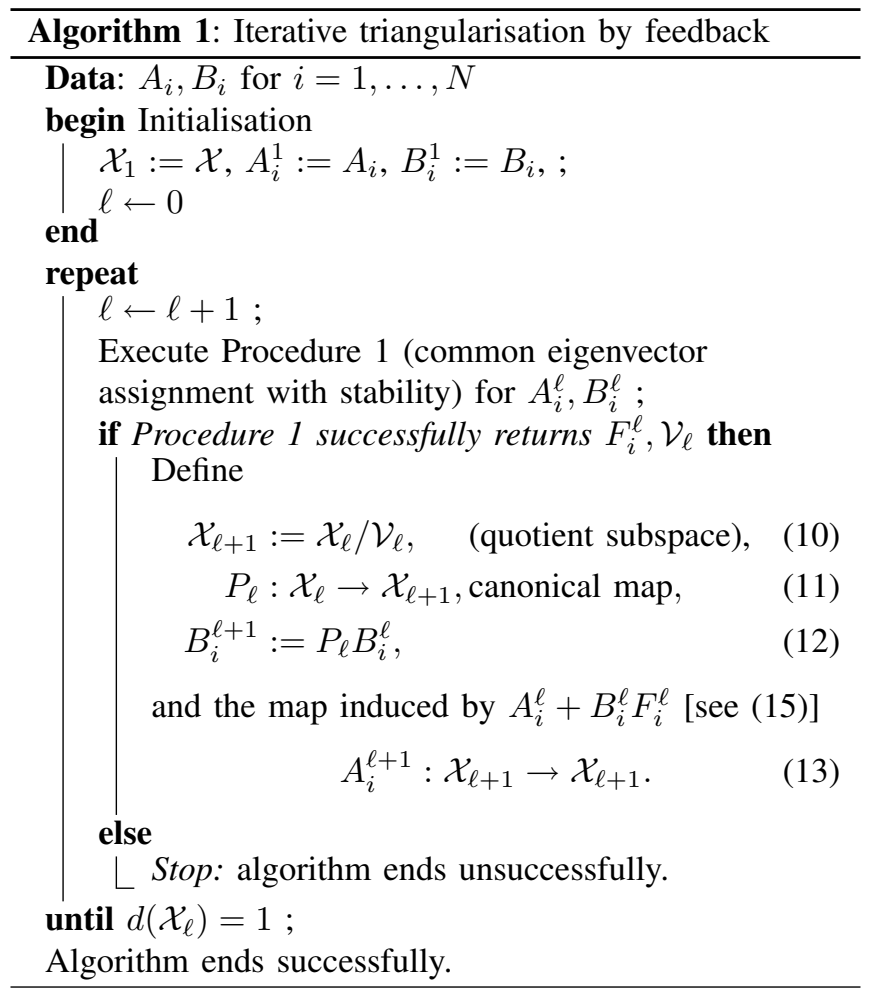

Some comments are in order before we state Theorem 1. Algorithm 1 begins by taking $\mathcal{X}_{1}=\mathcal{X}, A_{i}^{1}=A_{i}$ and $B_{i}^{1}=B_{i}$, and seeking feedback maps to assign a common eigenvector, with corresponding stable eigenvalues. If the common eigenvector assignment procedure (Procedure 1) is successful, then a one-dimensional subspace $\mathcal{V}_{1}$ is returned and note that, by (10), the dimension of $\mathcal{X}_{2}$ will satisfy $d\left(\mathcal{X}_{2}\right)=d\left(\mathcal{X}_{1}\right)-1$. This reduction in the dimension of the vector space considered occurs after every successful iteration of Procedure 1 until dimension one is reached.

When $d\left(\mathcal{X}_{\ell}\right)=1$, the maps $A_{i}^{\ell}+B_{i}^{\ell} F_{i}^{\ell}$ share the common eigenvector direction $\mathcal{V}_{\ell}=\mathcal{X}_{\ell}$ for any feedback map $F_{i}^{\ell}$, and hence Procedure 1 must necessarily return successfully at this point, after which Algorithm 1 terminates successfully. Therefore, the number of iterations required for successful termination of Algorithm 1 is $n$, the dimension of $\mathcal{X}$.

We now introduce a few more definitions required by Theorem 1. Let $\ell^{*}$ denote the last value of $\ell$ in Algorithm 1 for which Procedure 1 successfully computed the required maps $F_{i}^{\ell}$ and subspace $\mathcal{V}_{\ell}$. If Procedure 1 was never successful, define $\ell^{*}:=0$. Otherwise, $\ell^{*}>0$ and, from (12) and (13), we have for all $\ell=1, \ldots, \ell^{*}$ the maps

$$
A_{i}^{\ell}: \mathcal{X}_{\ell} \rightarrow \mathcal{X}_{\ell}, \quad B_{i}^{\ell}: \mathcal{U} \rightarrow \mathcal{X}_{\ell}
$$


and, by (10)-(13), the commutative diagram

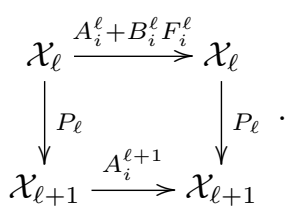

Finally, for all $\ell=1, \ldots, \ell^{*}$ and $i=1, \ldots, N$, we introduce the sequences of projections $\bar{P}_{\ell}: \mathcal{X} \rightarrow \mathcal{X}_{\ell+1}$, and state feedback maps $K_{i}^{\ell}: \mathcal{X} \rightarrow \mathcal{U}$ defined as

$$
\begin{aligned}
\bar{P}_{0} & :=\mathrm{I}, \quad \bar{P}_{\ell}:=\prod_{j=1}^{\ell} P_{j}=P_{\ell} P_{\ell-1} \cdots P_{1}, \\
K_{i}^{0} & :=0, \quad K_{i}^{\ell}:=\sum_{j=1}^{\ell} F_{i}^{j} \bar{P}_{j-1} .
\end{aligned}
$$

We are now ready to state Theorem 1, whose proof will be given in Section III-B.

Theorem 1 Problem 1 has a solution if and only if Algorithm 1 terminates successfully. In such a case, then $\ell^{*}=n$, the required feedback maps are given by

$$
K_{i}:=K_{i}^{n}
$$

with $K_{i}^{n}$ defined by (17), and the invariant flag (6)-(7) is given by

$$
\mathcal{S}_{j}:=\operatorname{ker} \bar{P}_{j}, \quad \text { for } j=1, \ldots, n
$$

If a solution to Problem 1 exists, Theorem 1 says that the required feedback maps and corresponding $A_{i}^{\mathrm{CL}}$-invariant flag can be found iteratively with Algorithm 1. On the other hand, if Algorithm 1 terminates successfully $\left(\ell^{*}=n\right)$, Theorem 1 guarantees the existence of a common quadratic control Lyapunov function for the switched system (as indicated following the statement of Problem 1).

The importance of Theorem 1 lies in the fact that it shows that the search for the desired stabilising feedback maps $K_{i}$ to solve Problem 1 can be performed iteratively by solving, at each iteration, a common eigenvector assignment problem. For autonomous systems, it is well-known that checking whether the Lie algebra generated by the subsystems evolution maps is solvable can be performed iteratively by seeking a common eigenvector and obtaining the induced map in a quotient subspace. However, to the best of the authors' knowledge, the fact that control design for stabilisation can also be performed in this iterative way has not been previously established.

The feedback maps that assign a common eigenvector with corresponding stable eigenvalues in Procedure 1 can be found numerically by posing and solving an optimisation problem. Due to space limitations, details on the numerical implementation of Procedure 1 will be reported elsewhere.

\section{B. Proof of Theorem 1}

The core of this section is the proof of Theorem 1. We require two preliminary lemmas, which are proved in the Appendix.

Lemma 2 Suppose that Algorithm 1 is executed and $\ell^{*}$ is the maximum value of $\ell$ reached for which Procedure 1 successfully computes $F_{i}^{\ell}$ and $\mathcal{V}_{\ell}$. Then

(i) $\bar{P}_{\ell}\left(A_{i}+B_{i} K_{i}^{\ell}\right)=A_{i}^{\ell+1} \bar{P}_{\ell}$, for $\ell=0, \ldots, \ell^{*}$, and

(ii) $K_{i}^{\ell^{*}} \mathcal{S}_{\ell}=K_{i}^{\ell} \mathcal{S}_{\ell}$, for $\ell=1, \ldots, \ell^{*}$ provided $\ell^{*}>0$.

(iii) $\bar{P}_{\ell}\left(A_{i}+B_{i} K_{i}^{\ell^{*}}\right) \mathcal{S}_{\ell+1}=\left(A_{i}^{\ell+1}+B_{i}^{\ell+1} F_{i}^{\ell+1}\right) \bar{P}_{\ell} \mathcal{S}_{\ell+1}$ for $\ell=0, \ldots, \ell^{*}-1$, provided $\ell^{*}>0$.

The next lemma is the key technical result employed in the necessity proof of Theorem 1 .

Lemma 3 Consider a set of maps $A_{i}^{\ell}: \mathcal{X}_{\ell} \rightarrow \mathcal{X}_{\ell}$ and $B_{i}^{\ell}$ : $\mathcal{U} \rightarrow \mathcal{X}_{\ell}$, for $i=1, \ldots, N$, and let $n_{\ell}:=d\left(\mathcal{X}_{\ell}\right)$. Suppose that there exist a set of maps $J_{i}^{\ell}: \mathcal{X}_{\ell} \rightarrow \mathcal{U}$ and a flag

$$
\mathcal{S}_{1}^{\ell} \subset \cdots \subset \mathcal{S}_{n_{\ell},}^{\ell}, \quad d\left(\mathcal{S}_{j}^{\ell}\right)=j
$$

that is invariant under $A_{i}^{\ell}+B_{i}^{\ell} J_{i}^{\ell}$, namely

$$
\begin{gathered}
\left(A_{i}^{\ell}+B_{i}^{\ell} J_{i}^{\ell}\right) \mathcal{S}_{j}^{\ell} \subset \mathcal{S}_{j}^{\ell}, \text { and } \\
\boldsymbol{\rho}\left(A_{i}^{\ell}+B_{i}^{\ell} J_{i}^{\ell}\right)<1,
\end{gathered}
$$

for $i=1, \ldots, N$ and $j=1, \ldots, n_{\ell}$. In addition, suppose that a set of maps $F_{i}^{\ell}: \mathcal{X}_{\ell} \rightarrow \mathcal{U}$ and a one-dimensional subspace $\mathcal{T}_{1} \subset \mathcal{X}_{\ell}$ with basis $\left\{t_{1}\right\}$ are known and satisfy

$$
\begin{gathered}
\left(A_{i}^{\ell}+B_{i}^{\ell} F_{i}^{\ell}\right) \mathcal{T}_{1} \subset \mathcal{T}_{1}, \text { and } \\
\boldsymbol{\rho}\left(A_{i}^{\ell}+B_{i}^{\ell} F_{i}^{\ell} \mid \mathcal{T}_{1}\right)<1,
\end{gathered}
$$

for $i=1, \ldots, N$. Then,

(i) There exist a set of maps $L_{i}^{\ell}: \mathcal{X}_{\ell} \rightarrow \mathcal{U}$ for $i=$ $1, \ldots, N$, and subspaces $\mathcal{T}_{2}, \ldots, \mathcal{T}_{n_{\ell}}$ satisfying, for $j=$ $1, \ldots, n_{\ell}$ and $i=1, \ldots, N$,

$$
\begin{gathered}
\mathcal{T}_{1} \subset \mathcal{T}_{2} \subset \cdots \subset \mathcal{T}_{n_{\ell}}=\mathcal{X}_{\ell}, \quad d\left(\mathcal{T}_{j}\right)=j, \\
\left(A_{i}^{\ell}+B_{i}^{\ell} L_{i}^{\ell}\right) \mathcal{T}_{j} \subset \mathcal{T}_{j} \\
\rho\left(A_{i}^{\ell}+B_{i}^{\ell} L_{i}^{\ell}\right)<1, \text { and } L_{i}^{\ell} t_{1}=F_{i}^{\ell} t_{1}
\end{gathered}
$$

(ii) Let $A_{i}^{\ell+1}: \mathcal{X}_{\ell} / \mathcal{T}_{1} \rightarrow \mathcal{X}_{\ell} / \mathcal{T}_{1}$ be the map induced by $A_{i}^{\ell}+B_{i}^{\ell} F_{i}^{\ell}$, let $P_{\ell}: \mathcal{X}_{\ell} \rightarrow \mathcal{X}_{\ell} / \mathcal{T}_{1}$ be the canonical projection, and let $B_{i}^{\ell+1}:=P_{\ell} B_{i}^{\ell}$, all for $i=1, \ldots, N$. Then, there exist a flag

$$
\mathcal{S}_{1}^{\ell+1} \subset \cdots \subset \mathcal{S}_{n_{\ell}-1}^{\ell+1}=\mathcal{X}_{\ell} / \mathcal{T}_{1}, \quad d\left(\mathcal{S}_{j}^{\ell+1}\right)=j,
$$

and maps $F_{i}^{\ell+1}: \mathcal{X}_{\ell} / \mathcal{T}_{1} \rightarrow \mathcal{U}$ such that

$$
\begin{aligned}
& \left(A_{i}^{\ell+1}+B_{i}^{\ell+1} F_{i}^{\ell+1}\right) \mathcal{S}_{j}^{\ell+1} \subset \mathcal{S}_{j}^{\ell+1}, \\
& \boldsymbol{\rho}\left(A_{i}^{\ell+1}+B_{i}^{\ell+1} F_{i}^{\ell+1}\right)<1,
\end{aligned}
$$

for $j=1, \ldots, n_{\ell}-1$ and $i=1, \ldots, N$.

We are now ready to present the proof of Theorem 1. Proof: (Theorem 1) 
Sufficiency: By (19) and (16), note that

$$
\mathcal{S}_{j} \subset \mathcal{S}_{j+1} \subset \mathcal{X}, \quad \text { for } j=1, \ldots, \ell^{*}-1,
$$

and

$$
d\left(\mathcal{S}_{j}\right)=j, \quad \text { for } j=1, \ldots, \ell^{*} .
$$

From Lemma 2(ii), we have

$$
\left(A_{i}+B_{i} K_{i}^{\ell^{*}}\right) \mathcal{S}_{j}=\left(A_{i}+B_{i} K_{i}^{j}\right) \mathcal{S}_{j} .
$$

Applying $\bar{P}_{j}$ to (33) and using Lemma 2(i),

$$
\bar{P}_{j}\left(A_{i}+B_{i} K_{i}^{j}\right) \mathcal{S}_{j}=A_{i}^{j+1} \bar{P}_{j} \mathcal{S}_{j}=0 .
$$

Equation (34) hence shows that $\left(A_{i}+B_{i} K_{i}^{j}\right) \mathcal{S}_{j} \subset \operatorname{ker} \bar{P}_{j}=$ $\mathcal{S}_{j}$, establishing (7).

Next, note that

$$
\mathcal{V}_{\ell}=\bar{P}_{\ell-1} \mathcal{S}_{\ell}
$$

With this observation, Lemma 2(iii) can be used inductively to show that

$$
\boldsymbol{\sigma}\left(A_{i}+B_{i} K_{i}\right)=\bigcup_{\ell=1}^{n} \boldsymbol{\sigma}\left(A_{i}^{\ell}+B_{i}^{\ell} F_{i}^{\ell} \mid \mathcal{V}_{\ell}\right)
$$

Then, $\rho\left(A_{i}+B_{i} K_{i}\right)<1$ follows from (9).

Since the dimension of $X_{\ell}$ is reduced by one at each iteration of $\ell$, and the dimension of $\mathcal{X}_{1}=\mathcal{X}$ is $n$, it takes Algorithm $1 n$ steps to terminate successfully, and so $\ell^{*}=n$. The sufficiency part of the proof is complete.

Necessity: The proof is by induction on $\ell$. Note first that Algorithm 1 terminates unsuccessfully if at some iteration there exist no feedback maps that can assign any common eigenvector. Since we assume that Problem 1 has a solution, then a flag (6) and feedback maps $K_{i}$ exist such that (7) is satisfied. This implies that, at the first iteration, $\ell=1$, a common assignable eigenvector indeed exists and the common eigenvector assignment procedure must return successfully.

Next, suppose that at iteration $\ell$ there exist a flag (20) in $\mathcal{X}_{\ell}$ and maps $J_{i}^{\ell}$ so that (21)-(22) are satisfied. Therefore, the common eigenvector assignment procedure can successfully compute $F_{i}^{\ell}$ and $\mathcal{V}_{\ell}$. Let $\mathcal{T}_{1}:=\mathcal{V}_{\ell}$ and, by (8), we have $\left(A_{i}^{\ell}+\right.$ $\left.B_{i}^{\ell} F_{i}^{\ell}\right) \mathcal{T}_{1} \subset \mathcal{T}_{1}$. Then, Lemma 3(i) establishes the existence of maps $L_{i}^{\ell}$ and subspaces $\mathcal{T}_{2}, \ldots, \mathcal{T}_{n}$ such that (25)-(27) are satisfied. Consequently, Lemma 3(ii) establishes that a flag (28) in $\mathcal{X}_{\ell+1}=\mathcal{X}_{\ell} / \mathcal{T}_{1}$ and feedback maps $F_{i}^{\ell+1}$ exist so that (29)-(30) are satisfied. This implies that at iteration $\ell+1$ the common eigenvector procedure (Procedure 1) terminates successfully. Finally, Algorithm 1 terminates successfully if and only if $\mathcal{X}_{\ell}$ is one-dimensional.

The proof of Theorem 1 is now complete.

\section{CONCLUSIONS}

This paper presents sufficient conditions for the uniform global exponential stabilisability of arbitrarily switched discrete-time linear systems with control inputs. These conditions establish the existence of a common quadratic control Lyapunov function for the system using Lie algebraic techniques in an iterative algorithm that solves a common eigenvector assignment problem at each iteration. It is shown that the Lie algebraic conditions that imply the existence of a CQLF for this problem are satisfied if and only if the proposed algorithm terminates successfully.

The approach proposed to solve this problem is restrictive, in that these Lie-algebraic conditions, which we seek to satisfy by feedback design, are only sufficient for the existence of a CQLF. However, a numerical implementation of the proposed algorithm can somewhat overcome these restrictions, in the sense that approximate numerical solutions may ensure stability even when a CQLF does not exist. Due to space limitations, details on such numerical implementation will be reported elsewhere.

\section{APPENDIX}

\section{Proof of Lemma 2}

(i): By induction on $\ell$. Note that (i) holds for $\ell=0$ by (16), (17), and the initialisation step of Algorithm 1. If $\ell^{*}=0$, then the result is already established. Next, in the case that $\ell^{*}>0$, suppose that (i) holds for some $\ell \geq 0$. We have

$$
\begin{aligned}
\bar{P}_{\ell+1}\left(A_{i}+B_{i} K_{i}^{\ell+1}\right) & \\
& =P_{\ell+1} \bar{P}_{\ell}\left(A_{i}+B_{i} K_{i}^{\ell}+B_{i} F_{i}^{\ell+1} \bar{P}_{\ell}\right),
\end{aligned}
$$

where we have used (16) and (17). Next, using (12) and our induction hypothesis, it follows from (37) that

$$
\bar{P}_{\ell+1}\left(A_{i}+B_{i} K_{i}^{\ell+1}\right)=P_{\ell+1}\left(A_{i}^{\ell+1}+B_{i}^{\ell+1} F_{i}^{\ell+1}\right) \bar{P}_{\ell} .
$$

Using (11) and (13) in (38), then

$$
\bar{P}_{\ell+1}\left(A_{i}+B_{i} K_{i}^{\ell+1}\right)=A_{i}^{\ell+2} P_{\ell+1} \bar{P}_{\ell}=A_{i}^{\ell+2} \bar{P}_{\ell+1},
$$

which establishes (i).

(ii): Using (16) and (17), it follows that

$$
\begin{array}{r}
K_{i}^{\ell^{*}} \mathcal{S}_{\ell}=\left(K_{i}^{\ell}+F_{i}^{\ell+1} \bar{P}_{\ell}+\ldots+F_{i}^{\ell^{*}} \bar{P}_{\ell^{*}-1}\right) \mathcal{S}_{\ell} \\
=K_{i}^{\ell} \mathcal{S}_{\ell},
\end{array}
$$

where the last line above follows from (19) and (16).

(iii): By induction on $\ell$. Using part (ii), (17) and the initialisation step of Algorithm 1, we have $\left(A_{i}+B_{i} K_{i}^{\ell^{*}}\right) \mathcal{S}_{1}=$ $\left(A_{i}+B_{i} K_{i}^{1}\right) \mathcal{S}_{1}=\left(A_{i}^{1}+B_{i}^{1} F_{i}^{1}\right) \mathcal{S}_{1}$. Recalling (16), it follows that (iii) is satisfied for $\ell=0$. Next, suppose that (iii) holds for some $0 \leq \ell<\ell^{*}-1$. From part (ii) and (17), we have

$$
\begin{aligned}
K_{i}^{\ell^{*}} \mathcal{S}_{\ell+2}=K_{i}^{\ell+2} \mathcal{S}_{\ell+2} & \\
= & \left(K_{i}^{\ell+1}+F_{i}^{\ell+2} \bar{P}_{\ell+1}\right) \mathcal{S}_{\ell+2},
\end{aligned}
$$

and using (41), part (i), (12), and (16), then

$$
\begin{aligned}
\bar{P}_{\ell+1}\left(A_{i}+B_{i} K_{i}^{\ell^{*}}\right) & \mathcal{S}_{\ell+2} \\
& =\left(A_{i}^{\ell+2}+B_{i}^{\ell+2} F_{i}^{\ell+2}\right) \bar{P}_{\ell+1} \mathcal{S}_{\ell+2},
\end{aligned}
$$

which establishes (iii) for $\ell+1$ and concludes the proof. 


\section{Proof of Lemma 3}

(i): Let $q:=\min j$ subject to $\mathcal{T}_{1} \subset \mathcal{S}_{j}^{\ell}$ and note that necessarily $1 \leq q \leq n_{\ell}$. Let $\left\{s_{1}, \ldots, s_{n_{\ell}}\right\}$ be a basis for $\mathcal{S}_{n_{\ell}}^{\ell}=\mathcal{X}_{\ell}$ such that $\left\{s_{1}, \ldots, s_{j}\right\}$ is a basis for $\mathcal{S}_{j}^{\ell}$, for $j=$ $1, \ldots, n_{\ell}$.

If $q=1$, it follows that $\mathcal{T}_{1}=\mathcal{S}_{1}^{\ell}$, and define $\mathcal{T}_{j}:=\mathcal{S}_{j}^{\ell}$ for $j=2, \ldots, n_{\ell}$. If $q>1$, define $\mathcal{T}_{j}:=\mathcal{T}_{1}+\mathcal{S}_{j-1}^{\ell}$ and note that $\mathcal{T}_{1} \cap \mathcal{S}_{j-1}^{\ell}=0$, for $j=2, \ldots, q$. Also, if $q<n_{\ell}$ define $\mathcal{T}_{j}:=\mathcal{S}_{j}^{\ell}$ for $j=q+1, \ldots, n_{\ell}$. Note that (25) is now established and that $\left\{t_{1}\right\} \cup\left(\left\{s_{1}, \ldots, s_{n_{\ell}}\right\} \backslash\left\{s_{q}\right\}\right)$ is a basis for $\mathcal{X}_{\ell}$.

Next, select $L_{i}^{\ell}$ such that, for $i=1, \ldots, N$,

$$
\begin{gathered}
L_{i}^{\ell} t_{1}=F_{i}^{\ell} t_{1}, \\
L_{i}^{\ell} s_{j}=J_{i}^{\ell} s_{j}, \text { for } j \in\left\{1, \ldots, n_{\ell}\right\} \backslash\{q\} .
\end{gathered}
$$

We next show that $\left(A_{i}^{\ell}+B_{i}^{\ell} L_{i}^{\ell}\right) \mathcal{T}_{j} \subset \mathcal{T}_{j}$. From (43), we have $\left(A_{i}^{\ell}+B_{i}^{\ell} L_{i}^{\ell}\right) t_{1}=\left(A_{i}^{\ell}+B_{i}^{\ell} F_{i}^{\ell}\right) t_{1}$ which, combined with (23) and (24) yields

$$
\begin{gathered}
\left(A_{i}^{\ell}+B_{i}^{\ell} L_{i}^{\ell}\right) \mathcal{T}_{1} \subset \mathcal{T}_{1}, \text { and } \\
\boldsymbol{\rho}\left(A_{i}^{\ell}+B_{i}^{\ell} L_{i}^{\ell} \mid \mathcal{T}_{1}\right)<1 .
\end{gathered}
$$

In addition, (44) implies that $\left(A_{i}^{\ell}+B_{i}^{\ell} L_{i}^{\ell}\right) s_{j}=\left(A_{i}^{\ell}+\right.$ $\left.B_{i}^{\ell} J_{i}^{\ell}\right) s_{j}$ for $j=1, \ldots, q-1$ which combined with (21) yields

$$
\left(A_{i}^{\ell}+B_{i}^{\ell} L_{i}^{\ell}\right) \mathcal{S}_{j} \subset \mathcal{S}_{j} \text { for } j=1, \ldots, q-1 .
$$

From (45), (47), and the definition of $\mathcal{T}_{j}$, it follows that

$$
\left(A_{i}^{\ell}+B_{i}^{\ell} L_{i}^{\ell}\right) \mathcal{T}_{j} \subset \mathcal{T}_{j}, \text { for } j=2, \ldots, q,
$$

which, if $q=n_{\ell}$, already establishes (26) for $j=1, \ldots, n_{\ell}$. If $q<n_{\ell}$, let $x \in \mathcal{S}_{j}^{\ell}=\mathcal{T}_{j}$ for some $q+1 \leq j \leq n_{\ell}$ and write $x=x_{1}+\sum_{p=q+1}^{j} c_{p} s_{p}$, with $x_{1} \in \mathcal{S}_{q}^{\ell}$. It follows that $\left(A_{i}^{\ell}+B_{i}^{\ell} L_{i}^{\ell}\right) x=\left(A_{i}^{\ell}+B_{i}^{\ell} L_{i}^{\ell}\right) x_{1}+\left(A_{i}^{\ell}+B_{i}^{\ell} J_{i}^{\ell}\right) \sum_{p=q+1}^{j} c_{p} s_{p}$ and from (48), (21) and the fact that $\mathcal{S}_{q}^{\ell}=\mathcal{T}_{q}$, then $\left(A_{i}^{\ell}+B_{i}^{\ell} L_{i}^{\ell}\right) x \in \mathcal{T}_{j}$. We have thus established (26) for $j=1, \ldots, n_{\ell}$.

Recall that $\mathcal{S}_{q}^{\ell}=\mathcal{T}_{q}=\mathcal{T}_{1} \oplus \mathcal{S}_{q-1}^{\ell}$, and consider the quotient space $\mathcal{X}_{\ell} / \mathcal{T}_{q}$. Let $\overline{A_{i}^{\ell}+B_{i}^{\ell} L_{i}^{\ell}}$ and $\overline{A_{i}^{\ell}+B_{i}^{\ell} J_{i}^{\ell}}$ be

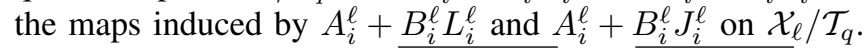
By (44), it follows that $\overline{A_{i}^{\ell}+B_{i}^{\ell} L_{i}^{\ell}}=\overline{A_{i}^{\ell}+B_{i}^{\ell} J_{i}^{\ell}}$ and that $\left(A_{i}^{\ell}+B_{i}^{\ell} L_{i}^{\ell}\right) \mathcal{S}_{q-1}^{\ell}=\left(A_{i}^{\ell}+B_{i}^{\ell} J_{i}^{\ell}\right) \mathcal{S}_{q-1}^{\ell} \subset \mathcal{S}_{q-1}^{\ell}$. From the latter facts and (45), we have $\sigma\left(A_{i}^{\ell}+B_{i}^{\ell} L_{i}^{\ell}\right)=$ $\boldsymbol{\sigma}\left(A_{i}^{\ell}+B_{i}^{\ell} L_{i}^{\ell} \mid \mathcal{T}_{1}\right) \cup \boldsymbol{\sigma}\left(A_{i}^{\ell}+B_{i}^{\ell} J_{i}^{\ell} \mid \mathcal{S}_{q-1}^{\ell}\right) \cup \boldsymbol{\sigma}\left(\overline{A_{i}^{\ell}+B_{i}^{\ell} J_{i}^{\ell}}\right)$, and recalling (22) and (46), then (27) is established and the proof of (i) is concluded.

(ii): Let $L_{i}^{\ell}$ and $\mathcal{T}_{2}, \ldots, \mathcal{T}_{n_{\ell}}$ be the maps and subspaces whose existence is asserted by part (i). Define $\mathcal{S}_{j}^{\ell+1}:=$ $P_{\ell} \mathcal{T}_{j+1}$ for $j=1, \ldots, n_{\ell}-1$, and note that by (25), then (28) is satisfied. We have

$$
\begin{aligned}
P_{\ell}\left(A_{i}^{\ell}+B_{i}^{\ell} L_{i}^{\ell}\right) & =P_{\ell}\left(A_{i}^{\ell}+B_{i}^{\ell} F_{i}^{\ell}+B_{i}^{\ell}\left(L_{i}^{\ell}-F_{i}^{\ell}\right)\right) \\
& =A_{i}^{\ell+1} P_{\ell}+B_{i}^{\ell+1}\left(L_{i}^{\ell}-F_{i}^{\ell}\right) .
\end{aligned}
$$

By (27), then $\mathcal{T}_{1} \subset \operatorname{ker}\left(L_{i}^{\ell}-F_{i}^{\ell}\right)$ and hence $L_{i}^{\ell}-F_{i}^{\ell}$ can be factored as

$$
L_{i}^{\ell}-F_{i}^{\ell}=F_{i}^{\ell+1} P_{\ell}
$$

Substituting (50) into (49) yields

$$
P_{\ell}\left(A_{i}^{\ell}+B_{i}^{\ell} L_{i}^{\ell}\right)=\left(A_{i}^{\ell+1}+B_{i}^{\ell+1} F_{i}^{\ell+1}\right) P_{\ell} .
$$

Combining (26) and (51), it follows that

$$
\begin{aligned}
P_{\ell}\left(A_{i}^{\ell}+B_{i}^{\ell} L_{i}^{\ell}\right) \mathcal{T}_{j+1}=\left(A_{i}^{\ell+1}+\right. & \left.B_{i}^{\ell+1} F_{i}^{\ell+1}\right) \mathcal{S}_{j}^{\ell+1} \\
& \subset P_{\ell} \mathcal{T}_{j+1}=\mathcal{S}_{j}^{\ell+1},
\end{aligned}
$$

for $j=1, \ldots, n_{\ell}-1$, whence (29) is established. Also, (30) follows from (51) and (27). This concludes the proof of (ii) and the proof of the lemma.

\section{REFERENCES}

[1] A.A. Agrachev and D. Liberzon. Lie-algebraic stability criteria for switched systems. SIAM J. Control and Optimization, 40(1):253-269, 2001.

[2] Z. Arstein. Stabilization with relaxed controls. Nonlinear Analysis, 7:1163-1173, 1983.

[3] G. Basile and G. Marro. Controlled and conditioned invariants in linear system theory. Prentice Hall, 1992.

[4] R.A. Decarlo, M.S. Branicky, S. Pettersson, and B. Lennartson. Perspectives and results on the stability and stabilizability of hybrid systems. Proc. of the IEEE, 88(7):1069-1082, 2000.

[5] K. Erdmann and M.J. Wildon. Introduction to Lie algebras. SpringerVerlag London, 2006.

[6] F. Felicioni and S. Junco. L'approche de l'algèbre de Lie pour l'adaptation des paramètres de la commande au changement de la période: stabilité, performance, et robustesse. In Conf. Internationale Francophone d'Automatique (CIFA), Bucarest, 2008. In French.

[7] F. Felicioni and S. Junco. A lie algebraic approach to design of stable feedback control systems with varying sampling rate. In IFAC World Congress, Seoul, South Korea, 2008.

[8] F. Felicioni, S. Junco, and H. Haimovich. Control de sistemas discretos LTI conmutados en base a métodos Lie-algebraicos, con aplicación al control sobre redes de comunicación. In Congr. Argentino de Control Automático (AADECA), Buenos Aires, Argentina, 2008. In Spanish.

[9] L. Gurvits. Stability of discrete linear inclusion. Linear Algebra and its Applications, 231:47-85, 1995.

[10] J. P. Hespanha. Modelling and analysis of stochastic hybrid systems. IEE Proceedings: Control Theory and Applications, 153(5):520-535, 2006.

[11] J.E. Humphreys. Introduction to Lie algebras and representation theory. Springer-Verlag New York, Heidelberg, Berlin, 1972.

[12] D. Liberzon. Switching in systems and control. Birkhäuser, 2003.

[13] D. Liberzon, J.P. Hespanha, and A.S. Morse. Stability of switched systems: a lie-algebraic condition. Systems and Control Letters, 37:117-122, 1999.

[14] D. Liberzon and S. Morse. Basic problems in stability and design of switched systems. Control Systems Magazine, October 1999.

[15] D. Liberzon and R. Tempo. Common lyapunov functions and gradient algorithms. IEEE Trans. on Automatic Control, 49(6):990-994, 2004.

[16] H. Lin and P. J. Antsaklis. Stability and stabilisability of switched linear systems: a survey of recent results. IEEE Trans. on Automatic Control, 54(2):308-322, February 2009.

[17] A. P. Molchanov and Ye. S. Pyatnitskiy. Criteria of asymptotic stability of differential and difference inclusions encountered in control theory. Systems and Control Letters, 13(1):59-64, 1989.

[18] A. S. Morse. Control using logic-based switching. In A. Isidori, editor, Trends in Control: a European perspective, chapter 2, pages 69-113. Springer, 1995.

[19] A. V. Savkin and R. J. Evans. Hybrid dynamical systems. Controller and sensor switching problems. Birkhuser, 2002.

[20] R. Shorten, F. Wirth, O. Mason, K. Wulff, and C. King. Stability criteria for switched and hybrid systems. SIAM Review, 49(4):545592, 2007.

[21] E.D. Sontag. A Lyapunov-like characterization of asymptotic controllability. SIAM J. on Control and Optimization, 21:462-471, 1983.

[22] J. Theys. Joint spectral radius: theory and approximations. PhD thesis, Center for Systems Engineering and Applied Mechanics, Université catholique de Louvain, 2005.

[23] W. M. Wonham. Linear multivariable control: a geometric approach Springer-Verlag, New York, 3rd edition, 1985. 